\title{
Existence results for sequential fractional integro-differential equations with nonlocal multi-point and strip conditions
}

\author{
Bashir Ahmad ${ }^{1 *}$, Sotiris K Ntouyas ${ }^{1,2}$, Ravi P Agarwal ${ }^{3}$ and Ahmed Alsaedi ${ }^{1}$
}

\section{"Correspondence:}

bashirahmad_qau@yahoo.com

${ }^{1}$ Nonlinear Analysis and Applied Mathematics (NAAM) - Research

Group, Department of Mathematics, Faculty of Science, King Abdulaziz University, P.O. Box 80203, Jeddah, 21589, Saudi Arabia

Full list of author information is available at the end of the article

\begin{abstract}
In this paper we investigate a new kind of nonlocal multi-point boundary value problem of Caputo type sequential fractional integro-differential equations involving Riemann-Liouville integral boundary conditions. Several existence and uniqueness results are obtained via suitable fixed point theorems. Some illustrative examples are also presented. The paper concludes with some interesting observations.
\end{abstract}

MSC: 34A08; 34B15

Keywords: sequential fractional derivative; multi-point; integral boundary conditions; existence; fixed point

\section{Introduction}

Fractional differential equations arise in many engineering and scientific disciplines such as physics, chemistry, aerodynamics, electrodynamics of complex medium or polymer rheology. In fact, the tools of fractional calculus have considerably improved the mathematical modeling of many real world problems. It has been mainly due to the fact that fractional-order operators provide an excellent instrument for the description of memory and hereditary properties of various materials and processes. For theoretical development and applications of the subject, we refer the reader to the books [1-3] and a series of papers [4-14], and the references cited therein.

Sequential fractional differential equations are also found to be of much interest [15, 16]. In fact, the concept of sequential fractional derivative is closely related to the nonsequential Riemann-Liouville derivatives; for details, see [17]. In [18], the authors studied different kinds of boundary value problems involving sequential fractional differential equations. In a recent article [19], the existence of solutions for higher-order sequential fractional differential inclusions with nonlocal three-point boundary conditions was discussed.

In this paper, we investigate the existence and uniqueness of solutions for a sequential fractional differential equation of the form

$$
\left({ }^{c} D^{q}+k^{c} D^{q-1}\right) x(t)=f\left(t, x(t),{ }^{c} D^{\beta} x(t), I^{\gamma} x(t)\right), \quad t \in[0,1], 2<q \leq 3,0<\beta, \gamma<1, k>0,
$$

\section{空 Springer}

(c) Ahmad et al. 2016. This article is distributed under the terms of the Creative Commons Attribution 4.0 International License (http://creativecommons.org/licenses/by/4.0/), which permits unrestricted use, distribution, and reproduction in any medium, provided you give appropriate credit to the original author(s) and the source, provide a link to the Creative Commons license, and indicate if changes were made. 
subject to nonlocal multi-point and Riemann-Liouville type integral boundary conditions:

$$
\begin{aligned}
& x(0)=0, \quad x^{\prime}(0)=0, \\
& \sum_{i=1}^{m} a_{i} x\left(\zeta_{i}\right)=\lambda \int_{0}^{\eta} \frac{(\eta-s)^{\delta-1}}{\Gamma(\delta)} x(s) d s, \quad \delta \geq 1,0<\eta<\zeta_{1}<\cdots<\zeta_{m}<1,
\end{aligned}
$$

where ${ }^{c} D^{(\cdot)}$ denotes the Caputo derivatives of fractional order $(\cdot), I^{(\cdot)}$ denotes the RiemannLiouville integral of fractional order $(\cdot), f:[0,1] \times \mathbb{R}^{3} \rightarrow \mathbb{R}$ is a given continuous function, and $\lambda, a_{i}, i=1, \ldots, m$ are real constants.

Here we emphasize that the coupling of nonlocal multi-point and Riemann-Liouville type strip condition considered on an arbitrary segment $(0, \eta) \subset[0,1]$ can be interpreted as the linear combination of the values of the unknown function at nonlocal points $\zeta_{i} \in(0,1)$ is proportional to the strip contribution of the unknown function. The consideration of the sequential fractional integro-differential equation (1.1) together with multi-point cum strip condition makes the problem (1.1)-(1.2) new.

The rest of the paper is arranged as follows. In Section 2, we establish a basic result that lays the foundation for defining a fixed point problem equivalent to the given problem (1.1)-(1.2). The main results, based on Banach's contraction mapping principle, Krasnoselskii's fixed point theorem and nonlinear alternative of Leray-Schauder type, are obtained in Section 3. Illustrating examples are discussed in Section 4, while Section 5 contains some interesting observations.

\section{Background material}

This section is devoted to some fundamental concepts of fractional calculus [20] and a basic lemma related to the linear variant of the given problem.

Definition 2.1 The fractional integral of order $\alpha$ with the lower limit zero for a function $\varphi$ is defined as

$$
I^{\alpha} \varphi(t)=\frac{1}{\Gamma(\alpha)} \int_{0}^{t} \frac{\varphi(s)}{(t-s)^{1-\alpha}} d s, \quad t>0, \alpha>0,
$$

provided the right-hand side is point-wise defined on $[0, \infty)$, where $\Gamma(\cdot)$ is the gamma function, which is defined by $\Gamma(\alpha)=\int_{0}^{\infty} t^{\alpha-1} e^{-t} d t$.

Definition 2.2 The Riemann-Liouville fractional derivative of order $\alpha>0, n-1<\alpha<n$, $n \in \mathbb{N}$, is defined as

$$
D_{0+}^{\alpha} \varphi(t)=\frac{1}{\Gamma(n-\alpha)}\left(\frac{d}{d t}\right)^{n} \int_{0}^{t}(t-s)^{n-\alpha-1} \varphi(s) d s, \quad t>0,
$$

where the function $\varphi$ has absolutely continuous derivative up to order $(n-1)$.

Definition 2.3 The Caputo derivative of order $\alpha$ for a function $\varphi:[0, \infty) \rightarrow \mathbb{R}$ can be written as

$$
{ }^{c} D^{\alpha} \varphi(t)=D_{0+}^{\alpha}\left(\varphi(t)-\sum_{k=0}^{n-1} \frac{t^{k}}{k !} \varphi^{(k)}(0)\right), \quad t>0, n-1<\alpha<n .
$$


Remark 2.4 If $\varphi(t) \in C^{n}[0, \infty)$, then

$$
{ }^{c} D^{\alpha} \varphi(t)=\frac{1}{\Gamma(n-\alpha)} \int_{0}^{t} \frac{\varphi^{(n)}(s)}{(t-s)^{\alpha+1-n}} d s=I^{n-\alpha} \varphi^{(n)}(t), \quad t>0, n-1<\alpha<n .
$$

To define the solution for problem (1.1)-(1.2), we consider the following lemma dealing with the linear variant of (1.1)-(1.2).

Lemma 2.1 For any $y \in C([0,1], \mathbb{R})$, a function $x \in C^{3}([0,1], \mathbb{R})$ is a solution of linear sequential fractional differential equation

$$
\left({ }^{c} D^{q}+k^{c} D^{q-1}\right) x(t)=y(t),
$$

subject to the boundary conditions (1.2) if and only if

$$
\begin{aligned}
x(t)= & \frac{k t-1+e^{-k t}}{\Delta}\left\{\lambda \int_{0}^{\eta} \frac{(\eta-s)^{\delta-1}}{\Gamma(\delta)}\left(\int_{0}^{s} e^{-k(s-\tau)}\left(\int_{0}^{\tau} \frac{(\tau-\omega)^{q-2}}{\Gamma(q-1)} y(\omega) d \omega\right) d \tau\right) d s\right. \\
& \left.-\sum_{i=1}^{m} a_{i} \int_{0}^{\zeta_{i}} e^{-k\left(\zeta_{i}-s\right)}\left(\int_{0}^{s} \frac{(s-\tau)^{q-2}}{\Gamma(q-1)} y(\tau) d \tau\right) d s\right\} \\
& +\int_{0}^{t} e^{-k(t-s)}\left(\int_{0}^{s} \frac{(s-\tau)^{q-2}}{\Gamma(q-1)} y(\tau) d \tau\right) d s,
\end{aligned}
$$

where

$$
\Delta=\sum_{i=1}^{m} a_{i}\left(k \zeta_{i}-1+e^{-k \zeta_{i}}\right)-\frac{\lambda}{\Gamma(\delta)}\left(\frac{k \eta^{\delta+1}}{\delta(\delta+1)}-\frac{\eta^{\delta}}{\delta}+\int_{0}^{\eta}(\eta-s)^{\delta-1} e^{-k s} d s\right) \neq 0 .
$$

Proof As argued in [18], the general solution of the equation (2.1) can be written as

$$
x(t)=b_{0} e^{-k t}+\frac{b_{1}}{k}\left(1-e^{-k t}\right)+\frac{b_{2}}{k^{2}}\left(k t-1+e^{-k t}\right)+\int_{0}^{t} e^{-k(t-s)}\left(\int_{0}^{s} \frac{(s-\tau)^{q-2}}{\Gamma(q-1)} y(\tau) d \tau\right) d s .
$$

Using the data $x(0)=0, x^{\prime}(0)=0$ given by (1.2) in (2.4), we find that $b_{0}=0$ and $b_{1}=0$. Thus (2.4) takes the form

$$
x(t)=\frac{b_{2}}{k^{2}}\left(k t-1+e^{-k t}\right)+\int_{0}^{t} e^{-k(t-s)}\left(\int_{0}^{s} \frac{(s-\tau)^{q-2}}{\Gamma(q-1)} y(\tau) d \tau\right) d s .
$$

Using the condition $\sum_{i=1}^{m} a_{i} x\left(\zeta_{i}\right)=\lambda \int_{0}^{\eta} \frac{(\eta-s)^{\delta-1}}{\Gamma(\delta)} x(s) d s$ in (2.5), we obtain

$$
\begin{aligned}
b_{2}= & \frac{k^{2}}{\Delta}\left\{\lambda \int_{0}^{\eta} \frac{(\eta-s)^{\delta-1}}{\Gamma(\delta)}\left(\int_{0}^{s} e^{-k(s-\tau)}\left(\int_{0}^{\tau} \frac{(\tau-\omega)^{q-2}}{\Gamma(q-1)} y(\omega) d \omega\right) d \tau\right) d s\right. \\
& \left.-\sum_{i=1}^{m} a_{i} \int_{0}^{\zeta_{i}} e^{-k\left(\zeta_{i}-s\right)}\left(\int_{0}^{s} \frac{(s-\tau)^{q-2}}{\Gamma(q-1)} y(\tau) d \tau\right) d s\right\}
\end{aligned}
$$


where $\Delta$ is given by (2.3). Substituting the value of $b_{2}$ in (2.5), we get the solution (2.2). The converse follows by direct computation. This completes the proof.

In the next lemma, we present some estimates that we need in the sequel.

Lemma 2.2 For $y \in C([0,1], \mathbb{R})$ with $\|y\|=\sup _{t \in[0,1]}|y(t)|$ we have

(i) $\left|\int_{0}^{\eta} \frac{(\eta-s)^{\delta-1}}{\Gamma(\delta)}\left(\int_{0}^{s} e^{-k(s-\tau)}\left(\int_{0}^{\tau} \frac{(\tau-\omega)^{q-2}}{\Gamma(q-1)} y(\omega) d \omega\right) d \tau\right) d s\right| \leq \frac{\eta^{q+\delta-2}}{k^{2} \Gamma(q) \Gamma(\delta)}\left(\eta k+e^{-k \eta}-1\right)\|y\|$.

(ii) $\left|\sum_{i=1}^{m} a_{i} \int_{0}^{\zeta_{i}} e^{-k\left(\zeta_{i}-s\right)}\left(\int_{0}^{s} \frac{(s-\tau)^{q-2}}{\Gamma(q-1)} y(\tau) d \tau\right) d s\right| \leq \sum_{i=1}^{m}\left|a_{i}\right| \zeta_{i}^{q-1}\left(1-e^{-k \zeta_{i}}\right) \frac{\|y\|}{k \Gamma(q)}$.

(iii) $\left|\int_{0}^{t} e^{-k(t-s)}\left(\int_{0}^{s} \frac{(s-\tau)^{q-2}}{\Gamma(q-1)} y(\tau) d \tau\right) d s\right| \leq \frac{1}{k \Gamma(q)}\left(1-e^{-k}\right)\|y\|$.

Proof (i) Obviously

$$
\int_{0}^{\tau} \frac{(\tau-\omega)^{q-2}}{\Gamma(q-1)} d \omega=\frac{\tau^{q-1}}{\Gamma(q)}
$$

and

$$
\int_{0}^{s} e^{-k(s-\tau)} \frac{\tau^{q-1}}{\Gamma(q)} d \tau \leq \frac{s^{q-1}}{\Gamma(q)} \int_{0}^{s} e^{-k(s-\tau)} d \tau=\frac{s^{q-1}}{k \Gamma(q)}\left(1-e^{-k s}\right) .
$$

Hence,

$$
\begin{aligned}
& \left|\int_{0}^{\eta} \frac{(\eta-s)^{\delta-1}}{\Gamma(\delta)}\left(\int_{0}^{s} e^{-k(s-\tau)}\left(\int_{0}^{\tau} \frac{(\tau-\omega)^{q-2}}{\Gamma(q-1)} y(\omega) d \omega\right) d \tau\right) d s\right| \\
& \quad \leq\|y\| \int_{0}^{\eta} \frac{(\eta-s)^{\delta-1}}{\Gamma(\delta)}\left(\frac{s^{q-1}}{k \Gamma(q)}\right)\left(1-e^{-k s}\right) d s \\
& \quad \leq\|y\| \frac{\eta^{\delta-1}}{\Gamma(\delta)}\left(\frac{\eta^{q-1}}{k \Gamma(q)}\right) \int_{0}^{\eta}\left(1-e^{-k s}\right) d s \leq \frac{\eta^{q+\delta-2}}{k^{2} \Gamma(\delta) \Gamma(q)}\left(\eta k+e^{-k \eta}-1\right)\|y\| .
\end{aligned}
$$

The proofs of (ii) and (iii) are similar. The proof is completed.

\section{Existence and uniqueness results}

This section is devoted to the main results concerning the existence and uniqueness of solutions for the problem (1.1)-(1.2). First of all, we fix our terminology.

Let $X=\left\{x: x \in C([0,1], \mathbb{R})\right.$ and $\left.{ }^{c} D^{\beta} x \in C([0,1], \mathbb{R})\right\}$ denotes the space equipped with the norm $\|x\|_{X}=\|x\|+\left\|{ }^{c} D^{\beta} x\right\|=\sup _{t \in[0,1]}|x(t)|+\left.\sup _{t \in[0,1]}\right|^{c} D^{\beta} x(t) \mid$. Observe that $\left(X,\|\cdot\|_{X}\right)$ is a Banach space.

Using Lemma 2.1, we introduce an operator $F: X \rightarrow X$ as follows:

$$
\begin{aligned}
F(x)(t)= & \frac{k t-1+e^{-k t}}{\Delta}\left\{\lambda \int _ { 0 } ^ { \eta } \frac { ( \eta - s ) ^ { \delta - 1 } } { \Gamma ( \delta ) } \left(\int_{0}^{s} e^{-k(s-\tau)}\right.\right. \\
& \left.\times\left(\int_{0}^{\tau} \frac{(\tau-\omega)^{q-2}}{\Gamma(q-1)} f\left(\omega, x(\omega),{ }^{c} D^{\beta} x(\omega), I^{\gamma} x(\omega)\right) d \omega\right) d \tau\right) d s \\
& \left.-\sum_{i=1}^{m} a_{i} \int_{0}^{\zeta_{i}} e^{-k\left(\zeta_{i}-s\right)}\left(\int_{0}^{s} \frac{(s-\tau)^{q-2}}{\Gamma(q-1)} f\left(\tau, x(\tau),{ }^{c} D^{\beta} x(\tau), I^{\gamma} x(\tau)\right) d \tau\right) d s\right\} \\
& +\int_{0}^{t} e^{-k(t-s)}\left(\int_{0}^{s} \frac{(s-\tau)^{q-2}}{\Gamma(q-1)} f\left(\tau, x(\tau),{ }^{c} D^{\beta} x(\tau), I^{\gamma} x(\tau)\right) d \tau\right) d s .
\end{aligned}
$$


Observe that problem (1.1)-(1.2) has solutions if the operator defined by (3.1) has fixed points.

For computational convenience, we set

$$
\begin{aligned}
& p=\sup _{t \in[0,1]}\left|\frac{\left(k t-1+e^{-k t}\right)}{\Delta}\right|=\frac{1}{|\Delta|}\left(e^{-k}+k-1\right), \\
& \bar{p}=\sup _{t \in[0,1]}\left|\frac{k\left(1-e^{-k t}\right)}{\Delta}\right|=\frac{1}{|\Delta|} k\left(1-e^{-k}\right), \\
& \Lambda=p \Delta_{1}+\frac{1}{k \Gamma(q)}\left(1-e^{-k}\right), \quad \Lambda_{1}=\bar{p} \Delta_{1}+\frac{1}{\Gamma(q)}\left(2-e^{-k}\right), \\
& L_{1}=1+\frac{1}{\Gamma(\gamma+1)}
\end{aligned}
$$

where

$$
\Delta_{1}=|\lambda| \frac{\eta^{q+\delta-2}}{k^{2} \Gamma(q) \Gamma(\delta)}\left(\eta k+e^{-k \eta}-1\right)+\sum_{i=1}^{m}\left|a_{i}\right| \zeta_{i}^{q-1}\left(1-e^{-k \zeta_{i}}\right) \frac{1}{k \Gamma(q)},
$$

and $\Delta$ is given by (2.3). Now the stage is set to present the uniqueness result.

Theorem 3.1 Let $f:[0,1] \times \mathbb{R}^{3} \rightarrow \mathbb{R}$ be a continuous function satisfying the condition

$\left(H_{1}\right)\left|f(t, x, y, z)-f\left(t, x_{1}, y_{1}, z_{1}\right)\right| \leq L\left[\left\|x-x_{1}\right\|+\left\|y-y_{1}\right\|+\left\|z-z_{1}\right\|\right]$,

for all $t \in[0,1], x, y, z, x_{1}, y_{1}, z_{1} \in \mathbb{R}$, where $L$ is the Lipschitz constant. Then the problem (1.1)-(1.2) has a unique solution if $L L_{1}\left(\Lambda+\frac{\Lambda_{1}}{\Gamma(2-\beta)}\right)<1$, where $\Lambda, \Lambda_{1}, L_{1}$ are given by (3.3).

Proof Let us fix

$$
r \geq \frac{M_{0}\left(\Lambda+\frac{\Lambda_{1}}{\Gamma(2-\beta)}\right)}{1-L L_{1}\left(\Lambda+\frac{\Lambda_{1}}{\Gamma(2-\beta)}\right)}
$$

where $\Lambda, \Lambda_{1}, L_{1}$ are given by (3.3) and $M_{0}=\sup _{t \in[0,1]}|f(t, 0,0,0)|$. Then we show that $F B_{r} \subset$ $B_{r}$ where

$$
B_{r}=\left\{x \in X:\|x\|_{X} \leq r\right\} .
$$

For $x \in B_{r}$, using $\left(H_{1}\right)$, we get

$$
\begin{aligned}
\left|f\left(t, x(t),{ }^{c} D^{\beta} x(t), I^{\gamma} x(t)\right)\right| & \leq\left|f\left(t, x(t),{ }^{c} D^{\beta} x(t), I^{\gamma} x(t)\right)-f(t, 0,0,0)\right|+|f(t, 0,0,0)| \\
& \leq L\left[|x(t)|+\left|{ }^{c} D^{\beta} x(t)\right|+\left|I^{\gamma} x(t)\right|\right]+M_{0} \\
& \leq L\left[\|x\|_{X}+\frac{1}{\Gamma(\gamma+1)}\|x\|\right]+M_{0} \\
& \leq L\left(1+\frac{1}{\Gamma(\gamma+1)}\right)\|x\|_{X}+M_{0} \\
& =L L_{1}\|x\|_{X}+M_{0} \leq L L_{1} r+M_{0} .
\end{aligned}
$$


Then, for $x \in X$, we have

$$
\begin{aligned}
|F(x)(t)| \leq & \sup _{t \in[0,1]}\left|\frac{k t-1+e^{-k t}}{\Delta}\right|\left\{| \lambda | \int _ { 0 } ^ { \eta } \frac { ( \eta - s ) ^ { \delta - 1 } } { \Gamma ( \delta ) } \left(\int_{0}^{s} e^{-k(s-\tau)}\right.\right. \\
& \left.\times\left(\int_{0}^{\tau} \frac{(\tau-\omega)^{q-2}}{\Gamma(q-1)}\left|f\left(\omega, x(\omega),{ }^{c} D^{\beta} x(\omega), I^{\gamma} x(\omega)\right)\right| d \omega\right) d \tau\right) d s \\
& \left.+\sum_{i=1}^{m}\left|a_{i}\right| \int_{0}^{\zeta_{i}} e^{-k\left(\zeta_{i}-s\right)}\left(\int_{0}^{s} \frac{(s-\tau)^{q-2}}{\Gamma(q-1)}\left|f\left(\tau, x(\tau),{ }^{c} D^{\beta} x(\tau), I^{\gamma} x(\tau)\right)\right| d \tau\right) d s\right\} \\
& +\int_{0}^{t} e^{-k(t-s)}\left(\int_{0}^{s} \frac{(s-\tau)^{q-2}}{\Gamma(q-1)}\left|f\left(\tau, x(\tau),{ }^{c} D^{\beta} x(\tau), I^{\gamma} x(\tau)\right)\right| d \tau\right) d s \\
\leq & \left(L L_{1} r+M_{0}\right)\left\{p \left[|\lambda| \frac{\eta^{q+\delta-2}}{k^{2} \Gamma(q) \Gamma(\delta)}\left(\eta k+e^{-k \eta}-1\right)\right.\right. \\
& \left.\left.+\sum_{i=1}^{m}\left|a_{i}\right| \zeta_{i}^{q-1}\left(1-e^{-k \zeta_{i}}\right) \frac{1}{k \Gamma(q)}\right]+\frac{1}{k \Gamma(q)}\left(1-e^{-k}\right)\right\} \\
\leq & \left(L L_{1} r+M_{0}\right) \Lambda,
\end{aligned}
$$

which, on taking the norm for $t \in[0,1]$, yields

$$
\|F x\| \leq\left(L L_{1} r+M_{0}\right) \Lambda
$$

Also we have

$$
\begin{aligned}
\left|F^{\prime}(x)(t)\right| \leq & \left.\left.\frac{k-k e^{-k t} \mid\left\{| \lambda | \int _ { 0 } ^ { \eta } \frac { ( \eta - s ) ^ { \delta - 1 } } { \Gamma ( \delta ) } \left(\int_{0}^{s} e^{-k(s-\tau)}\right.\right.}{}+\int_{0}^{\tau} \frac{(\tau-\omega)^{q-2}}{\Gamma(q-1)}\left|f\left(\omega, x(\omega),{ }^{c} D^{\beta} x(\omega), I^{\gamma} x(\omega)\right)\right| d \omega\right) d \tau\right) d s \\
& \left.+\sum_{i=1}^{m}\left|a_{i}\right| \int_{0}^{\zeta_{i}} e^{-k\left(\zeta_{i}-s\right)}\left(\int_{0}^{s} \frac{(s-\tau)^{q-2}}{\Gamma(q-1)}\left|f\left(\tau, x(\tau),{ }^{c} D^{\beta} x(\tau), I^{\gamma} x(\tau)\right)\right| d \tau\right) d s\right\} \\
& +k \int_{0}^{t} e^{-k(t-s)}\left(\int_{0}^{s} \frac{(s-\tau)^{q-2}}{\Gamma(q-1)}\left|f\left(\tau, x(\tau),{ }^{c} D^{\beta} x(\tau), I^{\gamma} x(\tau)\right)\right| d \tau\right) d s \\
& +\int_{0}^{t} \frac{(t-s)^{q-2}}{\Gamma(q-1)}\left|f\left(s, x(s),{ }^{c} D^{\beta} x(s), I^{\gamma} x(s)\right)\right| d s \\
\leq & \left(L L_{1} r+M_{0}\right)\left\{\overline { p } \left[|\lambda| \frac{\eta^{q+\delta-2}}{k^{2} \Gamma(q) \Gamma(\delta)}\left(\eta k+e^{-k \eta}-1\right)\right.\right. \\
& \left.\left.+\sum_{i=1}^{m}\left|a_{i}\right| \zeta_{i}^{q-1}\left(1-e^{-k \zeta_{i}}\right) \frac{1}{k \Gamma(q)}\right]+\frac{1}{\Gamma(q)}\left(2-e^{-k}\right)\right\} \\
\leq & \left(L L_{1} r+M_{0}\right) \Lambda_{1} .
\end{aligned}
$$

By the definition of the Caputo fractional derivative with $0<\beta<1$, we get

$$
\begin{aligned}
\left|{ }^{c} D^{\beta}(F x)(t)\right| & \leq \int_{0}^{t} \frac{(t-s)^{-\beta}}{\Gamma(1-\beta)}\left|F^{\prime}(x)(s)\right| d s \leq\left(L L_{1} r+M_{0}\right) \Lambda_{1} \int_{0}^{t} \frac{(t-s)^{-\beta}}{\Gamma(1-\beta)} d s \\
& \leq \frac{1}{\Gamma(2-\beta)}\left(L L_{1} r+M_{0}\right) \Lambda_{1} .
\end{aligned}
$$


Hence

$$
\|F(x)\|_{X}=\|F(x)\|+\left\|{ }^{c} D^{\beta} F(x)\right\| \leq\left(L L_{1} r+M_{0}\right) \Lambda+\frac{1}{\Gamma(2-\beta)}\left(L L_{1} r+M_{0}\right) \Lambda_{1}<r .
$$

This shows that $F$ maps $B_{r}$ into itself. Now, for $x, y \in X$ and for each $t \in[0,1]$, we obtain

$$
\begin{aligned}
& |(F x)(t)-(F y)(t)| \\
& \leq \sup _{t \in[0,1]}\left|\frac{k t-1+e^{-k t}}{\Delta}\right|\left\{| \lambda | \int _ { 0 } ^ { \eta } \frac { ( \eta - s ) ^ { \delta - 1 } } { \Gamma ( \delta ) } \left(\int_{0}^{s} e^{-k(s-\tau)}\right.\right. \\
& \times\left(\int_{0}^{\tau} \frac{(\tau-\omega)^{q-2}}{\Gamma(q-1)} \mid f\left(\omega, x(\omega),{ }^{c} D^{\beta} x(\omega), I^{\gamma} x(\omega)\right)\right. \\
& \left.\left.-f\left(\omega, y(\omega),{ }^{c} D^{\beta} y(\omega), I^{\gamma} y(\omega)\right) \mid d \omega\right) d \tau\right) d s \\
& +\sum_{i=1}^{m}\left|a_{i}\right| \int_{0}^{\zeta_{i}} e^{-k\left(\zeta_{i}-s\right)}\left(\int_{0}^{s} \frac{(s-\tau)^{q-2}}{\Gamma(q-1)} \mid f\left(\tau, x(\tau),{ }^{c} D^{\beta} x(\tau), I^{\gamma} x(\tau)\right)\right. \\
& \left.\left.-f\left(\tau, y(\tau),{ }^{c} D^{\beta} y(\tau), I^{\gamma} y(\tau)\right) \mid d \tau\right) d s\right\} \\
& +\int_{0}^{t} e^{-k(t-s)}\left(\int_{0}^{s} \frac{(s-\tau)^{q-2}}{\Gamma(q-1)} \mid f\left(\tau, x(\tau),{ }^{c} D^{\beta} x(\tau), I^{\gamma} x(\tau)\right)\right. \\
& \left.-f\left(\tau, x(\tau),{ }^{c} D^{\beta} x(\tau), I^{\gamma} x(\tau)\right) \mid d \tau\right) d s \\
& \leq L\left\{p\left[|\lambda| \frac{\eta^{q+\delta-2}}{k^{2} \Gamma(q) \Gamma(\delta)}\left(\eta k+e^{-k \eta}-1\right)+\sum_{i=1}^{m}\left|a_{i}\right| \zeta_{i}^{q-1}\left(1-e^{-k \zeta_{i}}\right) \frac{1}{k \Gamma(q)}\right]\right. \\
& \left.+\frac{1}{k \Gamma(q)}\left(1-e^{-k}\right)\right\}\left[\|x-y\|+\left\|D^{\beta} x-D^{\beta} y\right\|+\frac{1}{\Gamma(\gamma+1)}\|x-y\|\right] \\
& \leq L\left\{p\left[|\lambda| \frac{\eta^{q+\delta-2}}{k^{2} \Gamma(q) \Gamma(\delta)}\left(\eta k+e^{-k \eta}-1\right)+\sum_{i=1}^{m}\left|a_{i}\right| \zeta_{i}^{q-1}\left(1-e^{-k \zeta_{i}}\right) \frac{1}{k \Gamma(q)}\right]\right. \\
& \left.+\frac{1}{k \Gamma(q)}\left(1-e^{-k}\right)\right\}\left[\|x-y\|_{X}+\frac{1}{\Gamma(\gamma+1)}\|x-y\|\right] \\
& \leq L\left\{p\left[|\lambda| \frac{\eta^{q+\delta-2}}{k^{2} \Gamma(q) \Gamma(\delta)}\left(\eta k+e^{-k \eta}-1\right)+\sum_{i=1}^{m}\left|a_{i}\right| \zeta_{i}^{q-1}\left(1-e^{-k \zeta_{i}}\right) \frac{1}{k \Gamma(q)}\right]\right. \\
& \left.+\frac{1}{k \Gamma(q)}\left(1-e^{-k}\right)\right\}\left(1+\frac{1}{\Gamma(\gamma+1)}\right)\|x-y\|_{X} \\
& \leq L L_{1} \Lambda\|x-y\|_{X} \text {. }
\end{aligned}
$$

Also we have

$$
\left|(F x)^{\prime}(t)-(F y)^{\prime}(t)\right| \leq L L_{1} \Lambda_{1}\|x-y\|_{X}
$$


which implies that

$$
\begin{aligned}
\left|{ }^{c} D^{\beta} F(x)(t)-{ }^{c} D^{\beta} F(y)(t)\right| & \leq \int_{0}^{t} \frac{(t-s)^{-\beta}}{\Gamma(1-\beta)}\left|F^{\prime}(x)(s)-F^{\prime}(y)(s)\right| d s \\
& \leq \frac{L L_{1} \Lambda_{1}}{\Gamma(2-\beta)}\|x-y\|_{X} .
\end{aligned}
$$

From the above inequalities, we have

$$
\begin{aligned}
\|F(x)-F(y)\|_{X} & =\|F(x)-F(y)\|+\left\|{ }^{c} D^{\beta} F(x)-{ }^{c} D^{\beta} F(y)\right\| \\
& \leq L L_{1}\left(\Lambda+\frac{\Lambda_{1}}{\Gamma(2-\beta)}\right)\|x-y\|_{X} .
\end{aligned}
$$

As $L L_{1}\left(\Lambda+\frac{\Lambda_{1}}{\Gamma(2-\delta)}\right)<1, F$ is a contraction. Thus, the conclusion of the theorem follows by the contraction mapping principle. This completes the proof.

Now, we state a known result due to Krasnoselskii [21] which is needed to prove the existence of at least one solution of (1.1)-(1.2).

Theorem 3.2 Let $M$ be a closed, convex, bounded, and nonempty subset of a Banach space X. Let $\mathcal{G}_{1}, \mathcal{G}_{2}$ be the operators such that: (i) $\mathcal{G}_{1} x+\mathcal{G}_{2} y \in M$ whenever $x, y \in M$; (ii) $\mathcal{G}_{1}$ is compact and continuous; (iii) $\mathcal{G}_{2}$ is a contraction mapping. Then there exists $z \in M$ such that $z=\mathcal{G}_{1} z+\mathcal{G}_{2} z$.

Theorem 3.3 Assume that $f:[0,1] \times \mathbb{R}^{3} \rightarrow \mathbb{R}$ is a continuous function satisfying $\left(H_{1}\right)$. In addition, the following assumption holds:

$\left(H_{2}\right)\left|f\left(t, x_{1}, x_{2}, x_{3}\right)\right| \leq \mu(t), \forall\left(t, x_{1}, x_{2}, x_{3}\right) \in[0,1] \times \mathbb{R}^{3}$ with $\mu \in C\left([0,1], \mathbb{R}^{+}\right)$.

Then the boundary value problem (1.1)-(1.2) has at least one solution on [0,1] if

$$
L L_{1} p \Delta_{1}<1
$$

where $p$ is given by (3.2), and $L_{1}, \Delta_{1}$ are defined by (3.4).

Proof Letting $\sup _{t \in[0,1]}|\mu(t)|=\|\mu\|$, we fix

$$
r \geq\left(\Lambda+\frac{\Lambda_{1}}{\Gamma(2-\beta)}\right)\|\mu\|
$$

where $\Lambda, \Lambda_{1}$ are given by (3.3) and consider $\mathcal{B}_{r}=\left\{x \in X:\|x\|_{X} \leq r\right\}$. Define the operators $F_{1}$ and $F_{2}$ on $\mathcal{B}_{r}$ as

$$
\left(F_{1} x\right)(t)=\int_{0}^{t} e^{-k(t-s)}\left(\int_{0}^{s} \frac{(s-\tau)^{q-2}}{\Gamma(q-1)} f\left(\tau, x(\tau),{ }^{c} D^{\beta} x(\tau), I^{\gamma} x(\tau)\right) d \tau\right) d s
$$




$$
\begin{aligned}
\left(F_{2} x\right)(t)= & \frac{k t-1+e^{-k t}}{\Delta}\left\{\lambda \int _ { 0 } ^ { \eta } \frac { ( \eta - s ) ^ { \delta - 1 } } { \Gamma ( \delta ) } \left(\int_{0}^{s} e^{-k(s-\tau)}\right.\right. \\
& \left.\times\left(\int_{0}^{\tau} \frac{(\tau-\omega)^{q-2}}{\Gamma(q-1)} f\left(\omega, x(\omega),{ }^{c} D^{\beta} x(\omega), I^{\gamma} x(\omega)\right) d \omega\right) d \tau\right) d s \\
& \left.-\sum_{i=1}^{m} a_{i} \int_{0}^{\zeta_{i}} e^{-k\left(\zeta_{i}-s\right)}\left(\int_{0}^{s} \frac{(s-\tau)^{q-2}}{\Gamma(q-1)} f\left(\tau, x(\tau),{ }^{c} D^{\beta} x(\tau), I^{\gamma} x(\tau)\right) d \tau\right) d s\right\} .
\end{aligned}
$$

For $x, y \in \mathcal{B}_{r}$, using the notation (3.4), we have

$$
\left\|F_{1} x+F_{2} y\right\| \leq\left\{p \Delta_{1}+\frac{1}{k \Gamma(q)}\left(1-e^{-k}\right)\right\}\|\mu\|=\Lambda\|\mu\| .
$$

Also

$$
\left\|F_{1}^{\prime} x+F_{2}^{\prime} y\right\| \leq\left\{\bar{p} \Delta_{1}+\frac{1}{\Gamma(q)}\left(2-e^{-k}\right)\right\}\|\mu\|=\Lambda_{1}\|\mu\|,
$$

which implies that

$$
\begin{aligned}
\left|{ }^{c} D^{\beta}\left(F_{1} x+F_{2} y\right)\right| & \leq \int_{0}^{t} \frac{(t-s)^{-\beta}}{\Gamma(1-\beta)}\left|F_{1}^{\prime} x+F_{2}^{\prime} y\right| d s \\
& \leq \frac{\Lambda_{1}}{\Gamma(2-\beta)}\|\mu\| .
\end{aligned}
$$

From the above inequalities, we get

$$
\begin{aligned}
\left\|F_{1} x+F_{2} y\right\|_{X} & =\left\|F_{1} x+F_{2} y\right\|+\left\|{ }^{c} D^{\beta}\left(F_{1} x+F_{2} y\right)\right\| \\
& \leq\left(\Lambda+\frac{\Lambda_{1}}{\Gamma(2-\beta)}\right)\|\mu\|<r .
\end{aligned}
$$

Thus, $F_{1} x+F_{2} y \in \mathcal{B}_{r}$. In view of the condition (3.7), it can easily be shown that $F_{2}$ is a contraction. Note that continuity of $f$ implies that the operator $F_{1}$ is continuous. Also, $F_{1}$ is uniformly bounded on $\mathcal{B}_{r}$ as

$$
\begin{aligned}
& \left\|F_{1} x\right\| \leq \frac{\left(1-e^{-k}\right)\|\mu\|}{k \Gamma(q)}, \\
& \left\|F_{1}^{\prime} x\right\| \leq \frac{\left(2-e^{-k}\right)\|\mu\|}{\Gamma(q)}, \\
& \left\|{ }^{C} D^{\beta} F_{1} x\right\| \leq \frac{1}{\Gamma(2-\beta)} \frac{\left(2-e^{-k}\right)\|\mu\|}{\Gamma(q)},
\end{aligned}
$$

and

$$
\left\|F_{1} x\right\|_{X} \leq \frac{\left(1-e^{-k}\right)\|\mu\|}{k \Gamma(q)}+\frac{1}{\Gamma(2-\beta)} \frac{\left(2-e^{-k}\right)\|\mu\|}{\Gamma(q)} .
$$

Now we prove the compactness of the operator $F_{1}$. Setting $\Omega=[0,1] \times \mathcal{B}_{r} \times \mathcal{B}_{r} \times \mathcal{B}_{r}$, we define $\sup _{(t, x) \in \Omega}\left|f\left(t, x(t),{ }^{c} D^{\beta} x(t), I^{\gamma} x(t)\right)\right|=M_{r}$, and consequently, for $0<t_{1}<t_{2}<1$, we 
get

$$
\begin{aligned}
& \left|\left(F_{1} x\right)\left(t_{2}\right)-\left(F_{1} x\right)\left(t_{1}\right)\right| \\
& =\mid \int_{0}^{t_{2}} e^{-k\left(t_{2}-s\right)}\left(\int_{0}^{s} \frac{(s-u)^{q-2}}{\Gamma(q-1)} f\left(u, x(s),{ }^{c} D^{\beta} x(u), I^{\gamma} x(u)\right) d u\right) d s \\
& \quad-\int_{0}^{t_{1}} e^{-k\left(t_{1}-s\right)}\left(\int_{0}^{s} \frac{(s-u)^{q-2}}{\Gamma(q-1)} f\left(u, x(u),{ }^{c} D^{\beta} x(u), I^{\gamma} x(u)\right) d u\right) d s \mid \\
& \leq \frac{M_{r}}{k \Gamma(q)}\left(\left|t_{2}^{q}-t_{1}^{q}\right|+\left|t_{2}^{q} e^{-k t_{2}}-t_{1}^{q} e^{-k t_{1}}\right|\right)
\end{aligned}
$$

and

$$
\begin{aligned}
& \left|{ }^{c} D^{\beta} F_{1}(x)\left(t_{2}\right)-{ }^{c} D^{\beta} F_{1}(x)\left(t_{1}\right)\right| \\
& \quad \leq \int_{0}^{t_{1}} \frac{\left|\left(t_{1}-s\right)^{\beta}-\left(t_{2}-s\right)^{\delta}\right|}{\left(t_{1}-s\right)^{\beta}\left(t_{2}-s\right)^{\beta}}\left|F_{1}^{\prime}(x)(s)\right| d s+\int_{t_{1}}^{t_{2}}\left|\left(t_{2}-s\right)^{-\beta}\right|\left|F_{1}^{\prime}(x)(s)\right| d s \\
& \quad \leq \frac{1}{\Gamma(1-\beta)} \frac{\left(2-e^{-k}\right)}{\Gamma(q)}\left\{\int_{0}^{t_{1}} \frac{\left|\left(t_{1}-s\right)^{\beta}-\left(t_{2}-s\right)^{\beta}\right|}{\left(t_{1}-s\right)^{\beta}\left(t_{2}-s\right)^{\beta}} d s+\int_{t_{1}}^{t_{2}}\left|\left(t_{2}-s\right)^{-\beta}\right| d s\right\} .
\end{aligned}
$$

Clearly, $\left|F_{1}(x)\left(t_{2}\right)-F_{1}(x)\left(t_{1}\right)\right| \rightarrow 0$ and $\left|{ }^{c} D^{\beta} F_{1}(x)\left(t_{2}\right)-{ }^{c} D^{\beta} F_{1}(x)\left(t_{1}\right)\right| \rightarrow 0$ independent of $x$ as $t_{2} \rightarrow t_{1}$. Thus, $F_{1}$ is relatively compact on $\mathcal{B}_{r}$. Hence, by the Arzelá-Ascoli theorem, $F_{1}$ is compact on $\mathcal{B}_{r}$. Thus all the assumptions of Theorem 3.2 are satisfied and the conclusion of Theorem 3.2 implies that the boundary value problem (1.1)-(1.2) has at least one solution on $[0,1]$. This completes the proof.

Remark 3.4 In the above theorem we can interchange the roles of the operators $F_{1}$ and $F_{2}$ to obtain a second result replacing (3.7) by the following condition:

$$
\frac{L L_{1}}{k \Gamma(q)}\left(1-e^{-k}\right)<1 .
$$

In the next theorem, we prove the existence of solutions for the problem (1.1)-(1.2) via the Leray-Schauder nonlinear alternative.

Lemma 3.1 (Nonlinear alternative for single valued maps [22]) Let $E$ be a Banach space, $C$ a closed, convex subset of $E, U$ an open subset of $C$ and $0 \in U$. Suppose that $F: \bar{U} \rightarrow C$ is a continuous, compact (that is, $F(\bar{U})$ is a relatively compact subset of $C$ ) map. Then either

(i) F has a fixed point in $\bar{U}$, or

(ii) there is a $u \in \partial U$ (the boundary of $U$ in $C$ ) and $\lambda \in(0,1)$ with $u=\lambda F(u)$.

Theorem 3.5 Let $f:[0,1] \times \mathbb{R}^{3} \rightarrow \mathbb{R}$ be a continuous function and that

$\left(H_{3}\right)$ there exist a function $\phi \in C\left([0,1], \mathbb{R}^{+}\right)$, and a nondecreasing, subhomogeneous (that is, $\Omega(k x) \leq k \Omega(x)$ for all $k \geq 1$ and $\left.x \in \mathbb{R}^{+}\right)$function $\Omega: \mathbb{R}^{+} \rightarrow \mathbb{R}^{+}$such that $\left|f\left(t, x_{1}, x_{2}, x_{3}\right)\right| \leq$ $\phi(t) \Omega\left(\left\|x_{1}\right\|+\left\|x_{2}\right\|+\left\|x_{3}\right\|\right)$, for all $\left(t, x_{1}, x_{2}, x_{3}\right) \in[0,1] \times \mathbb{R}^{3} ;$

$\left(H_{4}\right)$ there exists a constant $M>0$ such that

$$
\frac{M}{\left(\Lambda+\frac{\Lambda_{1}}{\Gamma(2-\beta)}\right)\|\phi\| L_{1} \Omega(M)}>1,
$$

where $\Lambda, \Lambda_{1}$ and $L_{1}$ are given by (3.3). 
Then the boundary value problem (1.1)-(1.2) has at least one solution on [0,1].

Proof Consider the operator $F: X \rightarrow X$ defined by (3.1). In the first step, we show that $F$ maps bounded sets into bounded sets in $C([0,1], \mathbb{R})$. For a positive number $r$, let $\mathcal{B}_{r}=\{x \in$ $\left.C([0,1], \mathbb{R}):\|x\|_{X} \leq r\right\}$ be a bounded set in $C([0,1], \mathbb{R})$. Then

$$
\begin{aligned}
|F(x)(t)| \leq & \sup _{t \in[0,1]}\left|\frac{k t-1+e^{-k t}}{\Delta}\right|\left\{| \lambda | \int _ { 0 } ^ { \eta } \frac { ( \eta - s ) ^ { \delta - 1 } } { \Gamma ( \delta ) } \left(\int_{0}^{s} e^{-k(s-\tau)}\right.\right. \\
& \left.\times\left(\int_{0}^{\tau} \frac{(\tau-\omega)^{q-2}}{\Gamma(q-1)}\left|f\left(\omega, x(\omega),{ }^{c} D^{\beta} x(\omega), I^{\gamma} x(\omega)\right)\right| d \omega\right) d \tau\right) d s \\
& \left.+\sum_{i=1}^{m}\left|a_{i}\right| \int_{0}^{\zeta_{i}} e^{-k\left(\zeta_{i}-s\right)}\left(\int_{0}^{s} \frac{(s-\tau)^{q-2}}{\Gamma(q-1)}\left|f\left(\tau, x(\tau),{ }^{c} D^{\beta} x(\tau), I^{\gamma} x(\tau)\right)\right| d \tau\right) d s\right\} \\
& +\int_{0}^{t} e^{-k(t-s)}\left(\int_{0}^{s} \frac{(s-\tau)^{q-2}}{\Gamma(q-1)}\left|f\left(\tau, x(\tau),{ }^{c} D^{\beta} x(\tau), I^{\gamma} x(\tau)\right)\right| d \tau\right) d s \\
\leq & \sup _{t \in[0,1]}\left|\frac{k t-1+e^{-k t} \mid}{\Delta}\right|\left\{\lambda \mid \int_{0}^{\eta} \frac{(\eta-s)^{\delta-1}}{\Gamma(\delta)}\left(\int_{0}^{s} e^{-k(s-\tau)}\right.\right. \\
& \left.\times\left(\int_{0}^{\tau} \frac{(\tau-\omega)^{q-2}}{\Gamma(q-1)} \phi(\omega) \Omega\left(\|x\|+\left\|D^{\beta} x\right\|+\frac{1}{\Gamma(\gamma+1)}\|x\|\right) d \omega\right) d \tau\right) d s \\
& +\sum_{i=1}^{m}\left|a_{i}\right| \int_{0}^{\zeta_{i}} e^{-k\left(\zeta_{i}-s\right)} \\
& \left.\times\left(\int_{0}^{s} \frac{(s-\tau)^{q-2}}{\Gamma(q-1)} \phi(\tau) \Omega\left(\|x\|+\left\|D^{\beta} x\right\|+\frac{1}{\Gamma(\gamma+1)}\|x\|\right) d \tau\right) d s\right\} \\
& +\int_{0}^{t} e^{-k(t-s)}\left(\int_{0}^{s} \frac{(s-\tau)^{q-2}}{\Gamma(q-1)} \phi(\tau) \Omega\left(\|x\|+\left\|D^{\beta} x\right\|+\frac{1}{\Gamma(\gamma+1)}\|x\|\right) d \tau\right) d s \\
\leq & \left\{p \Delta_{1}+\frac{1}{k \Gamma(q)}\left(1-e^{-k}\right)\right\}\|\phi\| \Omega\left(L_{1}\|x\| X\right) \\
\leq & \Lambda\|\phi\| L_{1} \Omega\left(\|x\|_{X}\right),
\end{aligned}
$$

which, on taking the norm, for $t \in[0,1]$ yields

$$
\|F x\| \leq \Lambda\|\phi\| L_{1} \Omega\left(\|x\|_{X}\right)
$$

Also we have

$$
\begin{aligned}
\left|F^{\prime}(x)(t)\right| \leq & \left|\frac{k-k e^{-k t}}{\Delta}\right|\left\{| \lambda | \int _ { 0 } ^ { \eta } \frac { ( \eta - s ) ^ { \delta - 1 } } { \Gamma ( \delta ) } \left(\int_{0}^{s} e^{-k(s-\tau)}\right.\right. \\
& \left.\times\left(\int_{0}^{\tau} \frac{(\tau-\omega)^{q-2}}{\Gamma(q-1)}\left|f\left(\omega, x(\omega),{ }^{c} D^{\beta} x(\omega), I^{\gamma} x(\omega)\right)\right| d \omega\right) d \tau\right) d s \\
& \left.+\sum_{i=1}^{m}\left|a_{i}\right| \int_{0}^{\zeta_{i}} e^{-k\left(\zeta_{i}-s\right)}\left(\int_{0}^{s} \frac{(s-\tau)^{q-2}}{\Gamma(q-1)}\left|f\left(\tau, x(\tau),{ }^{c} D^{\beta} x(\tau), I^{\gamma} x(\tau)\right)\right| d \tau\right) d s\right\} \\
& +k \int_{0}^{t} e^{-k(t-s)}\left(\int_{0}^{s} \frac{(s-\tau)^{q-2}}{\Gamma(q-1)}\left|f\left(\tau, x(\tau),{ }^{c} D^{\beta} x(\tau), I^{\gamma} x(\tau)\right)\right| d \tau\right) d s
\end{aligned}
$$




$$
\begin{aligned}
& +\int_{0}^{t} \frac{(t-s)^{q-2}}{\Gamma(q-1)}\left|f\left(s, x(s),{ }^{c} D^{\beta} x(s), I^{\gamma} x(s)\right)\right| d s \\
\leq & \left\{\bar{p} \Delta_{1}+\frac{1}{\Gamma(q)}\left(2-e^{-k}\right)\right\}\|\phi\| \Omega\left(L_{1}\|x\|_{X}\right) \\
\leq & \Lambda_{1}\|\phi\| L_{1} \Omega\left(\|x\|_{X}\right) .
\end{aligned}
$$

By the definition of the Caputo fractional derivative with $0<\beta<1$, we get

$$
\begin{aligned}
\left|{ }^{c} D^{\beta}(F x)(t)\right| & \leq \int_{0}^{t} \frac{(t-s)^{-\beta}}{\Gamma(1-\beta)}\left|F^{\prime}(x)(s)\right| d s \\
& \leq \Lambda_{1}\|\phi\| L_{1} \Omega\left(\|x\|_{X}\right) \int_{0}^{t} \frac{(t-s)^{-\beta}}{\Gamma(1-\beta)} d s \\
& \leq \frac{1}{\Gamma(2-\beta)} \Lambda_{1}\|\phi\| L_{1} \Omega\left(\|x\|_{X}\right) .
\end{aligned}
$$

Hence

$$
\|F(x)\|_{X}=\|F(x)\|+\left\|{ }^{c} D^{\beta} F(x)\right\| \leq\left(\Lambda+\frac{\Lambda_{1}}{\Gamma(2-\beta)}\right)\|\phi\| L_{1} \Omega(r) .
$$

Next we show that $F$ maps bounded sets into equicontinuous sets of $C([0,1], \mathbb{R})$. Let $t_{1}, t_{2} \in[0,1]$ with $t_{1}<t_{2}$ and $x \in \mathcal{B}_{r}$, where $\mathcal{B}_{r}$ is a bounded set of $C([0,1], \mathbb{R})$. Then we obtain

$$
\begin{aligned}
\left|(F x)\left(t_{2}\right)-(F x)\left(t_{1}\right)\right| & \mid \\
\leq & \frac{k\left(t_{2}-t_{1}\right)+e^{-k t_{2}}-e^{-k t_{1}}}{\Delta}\left\{\lambda \int _ { 0 } ^ { \eta } \frac { ( \eta - s ) ^ { \delta - 1 } } { \Gamma ( \delta ) } \left(\int_{0}^{s} e^{-k(s-\tau)}\right.\right. \\
& \left.\times\left(\int_{0}^{\tau} \frac{(\tau-\omega)^{q-2}}{\Gamma(q-1)} f\left(\omega, x(\omega),{ }^{c} D^{\beta} x(\omega), I^{\gamma} x(\omega)\right) d \omega\right) d \tau\right) d s \\
& \left.-\sum_{i=1}^{m} a_{i} \int_{0}^{\zeta_{i}} e^{-k\left(\zeta_{i}-s\right)}\left(\int_{0}^{s} \frac{(s-\tau)^{q-2}}{\Gamma(q-1)} f\left(\tau, x(\tau),{ }^{c} D^{\beta} x(\tau), I^{\gamma} x(\tau)\right) d \tau\right) d s\right\} \mid \\
& +\mid \int_{0}^{t_{1}}\left(e^{-k\left(t_{2}-s\right)}-e^{-k\left(t_{1}-s\right)}\right)\left(\int_{0}^{s} \frac{(s-\tau)^{q-2}}{\Gamma(q-1)} f\left(\tau, x(\tau),{ }^{c} D^{\beta} x(\tau), I^{\gamma} x(\tau)\right) d \tau\right) d s \\
& +\int_{t_{1}}^{t_{2}} e^{-k\left(t_{2}-s\right)}\left(\int_{0}^{s} \frac{(s-\tau)^{q-2}}{\Gamma(q-1)} f\left(\tau, x(\tau),{ }^{c} D^{\beta} x(\tau), I^{\gamma} x(\tau)\right) d \tau\right) d s \mid \\
\leq & \mid \frac{k\left(t_{2}-t_{1}\right)+e^{-k t_{2}}-e^{-k t_{1}} \mid\left[|\lambda| \frac{\eta^{q+\delta-2}}{k^{2} \Gamma(q) \Gamma(\delta)}\left(\eta k+e^{-k \eta}-1\right)\right.}{\Delta} \\
& \left.+\sum_{i=1}^{m}\left|a_{i}\right| \zeta_{i}^{q-1}\left(1-e^{-k \zeta_{i}}\right) \frac{1}{k \Gamma(q)}\right]\|\phi\| L_{1} \Omega(r) \\
& +\mid \int_{0}^{t_{1}}\left(e^{-k\left(t_{2}-s\right)}-e^{-k\left(t_{1}-s\right)}\right)\left(\int_{0}^{s} \frac{(s-u)^{q-2}}{\Gamma(q-1)} d u\right) d s \\
& +\int_{t_{1}}^{t_{2}} e^{-k\left(t_{2}-s\right)}\left(\int_{0}^{s} \frac{(s-u)^{q-2}}{\Gamma(q-1)} d u\right) d s \mid\|\phi\| L_{1} \Omega(r) .
\end{aligned}
$$


Also

$$
\begin{aligned}
\mid{ }^{c} D^{\beta} & F(x)\left(t_{2}\right)-{ }^{c} D^{\beta} F(x)\left(t_{1}\right) \mid \\
& \leq \int_{0}^{t_{1}} \frac{\left|\left(t_{1}-s\right)^{\beta}-\left(t_{2}-s\right)^{\beta}\right|}{\left(t_{1}-s\right)^{\beta}\left(t_{2}-s\right)^{\beta}}\left|F^{\prime}(x)(s)\right| d s+\int_{t_{1}}^{t_{2}}\left|\left(t_{2}-s\right)^{-\beta}\right|\left|F^{\prime}(x)(s)\right| d s \\
& \leq \frac{\Lambda_{1}}{\Gamma(1-\beta)}\left\{\int_{0}^{t_{1}} \frac{\left|\left(t_{1}-s\right)^{\beta}-\left(t_{2}-s\right)^{\beta}\right|}{\left(t_{1}-s\right)^{\beta}\left(t_{2}-s\right)^{\beta}} d s+\int_{t_{1}}^{t_{2}}\left|\left(t_{2}-s\right)^{-\beta}\right| d s\right\}\|\phi\| L_{1} \Omega(r) .
\end{aligned}
$$

Obviously the right-hand side of the above inequalities tends to zero independently of $x \in \mathcal{B}_{r}$ as $t_{2}-t_{1} \rightarrow 0$. As $F$ satisfies the above assumptions, it follows by the Arzelá-Ascoli theorem that $F: C([0,1], \mathbb{R}) \rightarrow C([0,1], \mathbb{R})$ is completely continuous.

The result will follow from the Leray-Schauder nonlinear alternative (Lemma 3.1) once we have proved the boundedness of the set of all solutions to equations $x=\theta F x$ for $\theta \in$ $[0,1]$.

Let $x$ be a solution. Then, for $t \in[0,1]$, and using the computations in proving that $F$ is bounded, we have

$$
|x(t)| \leq\left\{p \Delta_{1}+\frac{1}{k \Gamma(q)}\left(1-e^{-k}\right)\right\}\|\phi\| \Omega\left(L_{1}\|x\|_{X}\right) \leq \Lambda\|\phi\| L_{1} \Omega\left(\|x\|_{X}\right),
$$

which, on taking the norm for $t \in[0,1]$ yields

$$
\|x\| \leq \Lambda\|\phi\| L_{1} \Omega\left(\|x\|_{X}\right)
$$

Also we have

$$
\left|x^{\prime}(t)\right| \leq\left\{\bar{p} \Delta_{1}+\frac{1}{\Gamma(q)}\left(2-e^{-k}\right)\right\}\|\phi\| \Omega\left(L_{1}\|x\|_{X}\right) \leq \Lambda_{1}\|\phi\| L_{1} \Omega\left(\|x\|_{X}\right) .
$$

By the definition of the Caputo fractional derivative with $0<\beta<1$, we get

$$
\left|{ }^{c} D^{\beta}(x)(t)\right| \leq \int_{0}^{t} \frac{(t-s)^{-\beta}}{\Gamma(1-\beta)}\left|x^{\prime}(s)\right| d s \leq \frac{\Lambda_{1}}{\Gamma(2-\beta)}\|\phi\| L_{1} \Omega\left(\|x\|_{X}\right) .
$$

Hence

$$
\|x\|_{X}=\|x\|+\left\|{ }^{c} D^{\delta} x\right\| \leq\left(\Lambda+\frac{\Lambda_{1}}{\Gamma(2-\beta)}\right)\|\phi\| L_{1} \Omega\left(\|x\|_{X}\right) .
$$

Consequently, we have

$$
\frac{\|x\|_{X}}{\left(\Lambda+\frac{\Lambda_{1}}{\Gamma(2-\beta)}\right)\|\phi\| L_{1} \Omega\left(\|x\|_{X}\right)} \leq 1
$$

In view of $\left(H_{4}\right)$, there exists $M$ such that $\|x\| \neq M$. Let us set

$$
U=\{x \in C([0,1], \mathbb{R}):\|x\|<M\}
$$


Note that the operator $F: \bar{U} \rightarrow C([0,1], \mathbb{R})$ is continuous and completely continuous. From the choice of $U$, there is no $x \in \partial U$ such that $x=\theta F(x)$ for some $\theta \in(0,1)$. Consequently, by the nonlinear alternative of Leray-Schauder type (Lemma 3.1), we deduce that $F$ has a fixed point $x \in \bar{U}$ which is a solution of the problem (1.1)-(1.2). This completes the proof.

\section{Examples}

Consider the following nonlocal multi-point boundary value problem of the Caputo type sequential fractional integro-differential equations:

$$
\left\{\begin{array}{l}
\left({ }^{c} D^{8 / 3}+\frac{2}{3}{ }^{c} D^{5 / 3}\right) x(t)=f\left(t, x(t),{ }^{c} D^{3 / 4} x(t), I^{1 / 2} x(t)\right), \quad 0<t<1, \\
x(0)=0, x^{\prime}(0)=0, \quad x\left(\frac{1}{5}\right)+\frac{3}{2} x\left(\frac{2}{5}\right)+\frac{5}{2} x\left(\frac{3}{5}\right)+3 x\left(\frac{4}{5}\right)=\int_{0}^{\frac{1}{10}} \frac{\left(\frac{1}{10}-s\right)^{\frac{1}{2}}}{\Gamma\left(\frac{3}{2}\right)} x(s) d s .
\end{array}\right.
$$

Here $q=8 / 3, k=2 / 3, \beta=3 / 4, \gamma=1 / 2, a_{1}=1, a_{2}=3 / 2, a_{3}=5 / 2, a_{4}=3, \zeta_{i}=i / 5, i=1, \ldots, 4$, $\lambda=1, \eta=1 / 10$. With the given values, it is found that $\Delta \approx 0.6148915, \Delta_{1} \approx 1.287595, p \approx$ $1.648123, \bar{p} \approx 0.527554, \Lambda \approx 2.607218, \Lambda_{1} \approx 1.667317, L_{1} \approx 2.128379$. Now we illustrate the obtained results by choosing different values of $f\left(t, x(t),{ }^{c} D^{3 / 4} x(t), I^{1 / 2} x(t)\right)$. Let us first consider

$$
\begin{aligned}
f\left(t, x(t),{ }^{c} D^{3 / 4} x(t), I^{1 / 2} x(t)\right)= & \frac{1}{\sqrt{t+121}}\left(\frac{|x(t)|}{1+|x(t)|}+\tan ^{-1}\left({ }^{c} D^{3 / 4} x(t)\right)\right) \\
& +\frac{1}{11} I^{1 / 2} x(t)+\cos (\pi t / 2) .
\end{aligned}
$$

Obviously $L=1 / 11$ as $\left|f\left(t, x(t),{ }^{c} D^{3 / 4} x(t), I^{1 / 2} x(t)\right)-f\left(t, y(t),{ }^{c} D^{3 / 4} y(t), I^{1 / 2} y(t)\right)\right| \leq \frac{1}{11}(\|x-y\|+$ $\left.\left\|{ }^{c} D^{3 / 4} x-{ }^{c} D^{3 / 4} y\right\|+\left\|I^{1 / 2} x-I^{1 / 2} y\right\|\right)$. Further, $L L_{1}\left(\Lambda+\frac{\Lambda_{1}}{\Gamma(2-\beta)}\right) \approx 0.860389<1$. Thus all the conditions of Theorem 3.1 are satisfied. Therefore, by the conclusion of Theorem 3.1, we conclude that there exists a unique solution for the problem $(4.1)$ on $[0,1]$.

Next we show the applicability of Theorem 3.3 with the nonlinear function $f$ given by

$$
f\left(t, x(t),{ }^{c} D^{3 / 4} x(t), I^{1 / 2} x(t)\right)=\frac{3}{t+20}\left(\sin (x(t))+\frac{\left|{ }^{c} D^{3 / 4} x(t)\right|}{1+\left|{ }^{c} D^{3 / 4} x(t)\right|}\right)+\frac{3}{20} I^{1 / 2} x(t)+\frac{1}{10},
$$

with $|x(t)| \leq \varrho, t \in[0,1]$ ( $\varrho$ is a real constant). In this case $\mu(t)=\frac{6}{t+20}+\frac{3 \varrho}{10 \sqrt{\pi}}+\frac{1}{10}, L=3 / 20$ and $L L_{1} p \Delta_{1} \approx 0.6775$. Clearly all the conditions of Theorem 3.3 hold true. In consequence, the conclusion of Theorem 3.3 implies that the problem (4.1) with the given value of $f$ has at least one solution on $[0,1]$.

Finally, for the applicability of Theorem 3.5 , we choose

$$
f\left(t, x(t),{ }^{c} D^{3 / 4} x(t), I^{1 / 2} x(t)\right)=\frac{1}{40+t}\left(x(t) \cos (x(t))+{ }^{c} D^{3 / 4} x(t)+\frac{\sqrt{\pi}}{2} I^{1 / 2} x(t)+2\right) .
$$

It is easy to see that $\left|f\left(t, x(t),{ }^{c} D^{3 / 4} x(t), I^{1 / 2} x(t)\right)\right| \leq(2 /(40+t))\left(\|x\|_{X}+1\right)$. Then, by the condition $\left(H_{4}\right)$, with $\Omega\left(\|x\|_{X}\right)=1+\|x\|_{X}$ and $\|\phi\|=1 / 20$, we find that $M>M_{1} \approx 0.898304$. As all the conditions of Theorem 3.5 are satisfied, so it follows by its conclusion that there exists at least one solution for the problem (4.1) with the chosen value of $f$. 


\section{Conclusions}

We have discussed the existence and uniqueness of solutions for sequential fractional integro-differential equations involving the Caputo (Liouville-Caputo) derivative supplemented with nonlocal multi-point boundary conditions coupled with Riemann-Liouville type strip condition. Our results are not only new in the given configuration but also correspond to some new situations associated with the specific values of the parameters involved in the given problem. For example, our results correspond to the multi-point boundary conditions with classical nonlocal strip condition: $\sum_{i=1}^{m} a_{i} x\left(\zeta_{i}\right)=\lambda \int_{0}^{\eta} x(s) d s$ if we take $\delta=1$ in (1.2). In the case we choose $a_{i}=0, i=1, \ldots,(m-1), a_{m}=1$, and $\zeta_{m} \rightarrow 1$, our results correspond to the condition $x(1)=\lambda \int_{0}^{\eta} \frac{(\eta-s)^{\delta-1}}{\Gamma(\delta)} x(s) d s$.

\section{Competing interests}

The authors declare that they have no competing interests.

\section{Authors' contributions}

Each of the authors, BA, SKN, RPA, and AA, contributed to each part of this work equally and read and approved the final version of the manuscript.

\section{Author details}

${ }^{1}$ Nonlinear Analysis and Applied Mathematics (NAAM) - Research Group, Department of Mathematics, Faculty of Science, King Abdulaziz University, P.O. Box 80203, Jeddah, 21589, Saudi Arabia. ${ }^{2}$ Department of Mathematics, University of loannina, loannina, 451 10, Greece. ${ }^{3}$ Department of Mathematics, Texas A\&M University, Kingsville, TX 78363-8202, USA.

\section{Acknowledgements}

We thank the reviewers for their useful remarks that led to the improvement of our work.

Received: 24 September 2016 Accepted: 8 November 2016 Published online: 22 November 2016

\section{References}

1. Kilbas, AA, Srivastava, HM, Trujillo, JJ: Theory and Applications of Fractional Differential Equations. North-Holland Mathematics Studies, vol. 204. Elsevier Science B.V., Amsterdam (2006)

2. Magin, RL: Fractional Calculus in Bioengineering. Begell House Publishers Inc., US (2006)

3. Klafter, J, Lim, SC, Metzler, R (eds.): Fractional Dynamics in Physics. World Scientific, Singapore (2012)

4. Konjik, S, Oparnica, L, Zorica, D: Waves in viscoelastic media described by a linear fractional model. Integral Transforms Spec. Funct. 22, 283-291 (2011)

5. Balachandran, K, Divya, S, Rivero, M, Trujillo, JJ: Controllability of nonlinear implicit neutral fractional Volterra integrodifferential systems. J. Vib. Control 22, 2165-2172 (2016)

6. Ahmad, B, Agarwal, RP: Some new versions of fractional boundary value problems with slit-strips conditions. Bound. Value Probl. 2014, 175 (2014)

7. Li, Y, Qi, A: Positive solutions for multi-point boundary value problems of fractional differential equations with p-Laplacian. Math. Methods Appl. Sci. 39, 1425-1434 (2016)

8. Liang, $S$, Zhang, J: Existence of multiple positive solutions for $m$-point fractional boundary value problems on an infinite interval. Math. Comput. Model. 54, 1334-1346 (2011)

9. Bai, ZB, Sun, W: Existence and multiplicity of positive solutions for singular fractional boundary value problems. Comput. Math. Appl. 63, 1369-1381 (2012)

10. Cabada, A, Wang, G: Positive solutions of nonlinear fractional differential equations with integral boundary value conditions. J. Math. Anal. Appl. 389, 403-411 (2012)

11. Zhang, L, Wang, G, Ahmad, B, Agarwal, RP: Nonlinear fractional integro-differential equations on unbounded domains in a Banach space. J. Comput. Appl. Math. 249, 51-56 (2013)

12. O'Regan, D, Stanek, S: Fractional boundary value problems with singularities in space variables. Nonlinear Dyn. 71, 641-652 (2013)

13. Tariboon, J, Sitthiwirattham, T, Ntouyas, SK: Existence results for fractional differential inclusions with multi-point and fractional integral boundary conditions. J. Comput. Anal. Appl. 17, 343-360 (2014)

14. Ahmad, B, Nieto, JJ: A class of differential equations of fractional order with multi-point boundary conditions. Georgian Math. J. 21, 243-248 (2014)

15. Klimek, M: Sequential fractional differential equations with Hadamard derivative. Commun. Nonlinear Sci. Numer. Simul. 16, 4689-4697 (2011)

16. Bai, C: Impulsive periodic boundary value problems for fractional differential equation involving Riemann-Liouville sequential fractional derivative. J. Math. Anal. Appl. 384, 211-231 (2011)

17. Miller, KS, Ross, B: An Introduction to the Fractional Calculus and Fractional Differential Equations. Wiley and Sons, New York (1993)

18. Ahmad, B, Nieto, JJ: Boundary value problems for a class of sequential integrodifferential equations of fractional order. J. Funct. Spaces Appl. 2013, Article ID 149659 (2013)

19. Ahmad, B, Ntouyas, SK: On higher-order sequential fractional differential inclusions with nonlocal three-point boundary conditions. Abstr. Appl. Anal. 2014, Article ID 659405 (2014) 
20. Zhou, Y: Basic Theory of Fractional Differential Equations. World Scientific Publishing Co. Pte. Ltd., Hackensack (2014)

21. Smart, DR: Fixed Point Theorems. Cambridge University Press, Cambridge (1980)

22. Granas, A, Dugundji, J: Fixed Point Theory. Springer, New York (2003)

Submit your manuscript to a SpringerOpen ${ }^{\circ}$ journal and benefit from:

- Convenient online submission

Rigorous peer review

- Immediate publication on acceptance

- Open access: articles freely available online

- High visibility within the field

- Retaining the copyright to your article

Submit your next manuscript at $>$ springeropen.com 